\title{
Some properties of eigenvalues and eigenfunctions of the cubic oscillator with imaginary coupling constant
}

\author{
G. Andrei Mezincescu† $\ddagger$ \\ †INFM, C.P. MG-7, R-76900 Bucureşti - Măgurele, România, and \\ Centrul de Cercetări Avansate de Fizică al Academiei Române, Bucureşti, România
}

\begin{abstract}
Comparison between the exact value of the spectral zeta function, $Z_{H}(1)=5^{-6 / 5}[3-2 \cos (\pi / 5)] \Gamma^{2}(1 / 5) / \Gamma(3 / 5)$, and the results of numeric and WKB calculations supports the conjecture by Bessis that all the eigenvalues of this PTinvariant hamiltonian are real. For one-dimensional Schrödinger operators with complex potentials having a monotonic imaginary part, the eigenfunctions (and the imaginary parts of their logarithmic derivatives) have no real zeros. PACS numbers:

03.65.Db, 02.30.Tb, 02.60.Lj, 11.30.Er
\end{abstract}

\section{Introduction}

Some years ago Bessis (1995) had conjectured that all the eigenvalues of the cubic oscillator with purely imaginary coupling constant,

$$
H=-\frac{\mathrm{d}^{2}}{\mathrm{~d} x^{2}}+\mathrm{i} g x^{3} ; \quad \operatorname{Im}(g)=0,
$$

were real. Numerical and WKB investigations have found only real eigenvalues (Bessis, 1995, Bender and Boettcher 1998ab, Bender et al 1999), although a rigorous proof is still lacking.

The operator (值), defined on the intersection of the domains of $\mathrm{d}^{2} / \mathrm{d} x^{2}$ and $x^{3}$ is closed and has a compact, Hilbert-Schmidt resolvent (Caliceti et al 1980, Caliceti 1999). Thus, its spectrum consists only of eigenvalues, which can acumulate only near infinity. Since its numerical range lies in the right complex half-plane $[\operatorname{Re}(z)>0]$, the real parts of the eigenvalues are positive (Kato 1966).

A Schrödinger operator

$$
H=-\frac{\mathrm{d}^{2}}{\mathrm{~d} x^{2}}+U(x)
$$

is called PT-invariant if the potential satisfies

$$
U(x)=\overline{U(-x)} \text {. }
$$

Here, and in the following, we use the overline to denote complex conjugation. Although (1) is not Hermitean (symmetric), it is PT-invariant, but not separately

‡ E-mail: mezin@alpha1.infim.ro 
invariant under the spatial inversion $\mathrm{P},[\phi(x) \rightarrow \phi(-x)]$, or the time reversal $\mathrm{T}$, $[\phi(x) \rightarrow \overline{\phi(x)}]$, which map it on its adjoint $H^{*}$.

Adding harmonic term to the potential in (11), we obtain

$$
U(x)=\mathrm{i} g x^{3}+\mu x^{2} ; \quad \mu \geq 0 .
$$

The PT-invariant operator defined by (2) and (4) has been intensely investigated. Caliceti et al (1980) have shown that each eigenvalue of the harmonic oscillator (to which it reduces for $g=0$ ) stays real for sufficiently small real $g$ or, equivalently, for sufficiently large $\mu$ (Delabaere and Pham 1999).

The resolvent $(z-H)^{-1}$ of the one-dimensional Schrdinger operator (2) is an integral operator for all $z$ in the resolvent set. Its kernel, $G_{z}(x, y)$ can be expressed through 2 solutions that satisfy the boundary condition at only one end of the interval (Naimark 1964, Caliceti et al 1980). Let $f_{+}(x ; z)\left[f_{-}(x ; z)\right]$ be the (unique, up to a multiplicative constant) solution of the equation

$$
(z-H) f=f^{\prime \prime}+[z-U(x)] f=0,
$$

which, together with its first derivative, is locally $L^{2}$ near $+\infty$ (respectively $-\infty$ ). One can readily check that the kernel is

$$
G_{z}(x, y)=\frac{\theta(x-y) f_{+}(x ; z) f_{-}(y, z)+\theta(y-x) f_{+}(y ; z) f_{-}(x ; z)}{W\left[f_{+}, f_{-}\right](z)} .
$$

Here $\theta(x)$ is the Heaviside function (characteristic function of the positive half-axis), and

$$
W\left[f_{+}, f_{-}\right](z)=f_{+}^{\prime}(x ; z) f_{-}(x ; z)-f_{-}^{\prime}(x ; z) f_{+}(x ; z),
$$

is the ( $x$-independent) Wronskian of $f_{+}$and $f_{-}$.

Generally speaking, a PT-invariant Schrödinger operator (2), (3) can have any kind of spectrum, as long as it is invariant with respect to complex conjugation. For example, let

$$
U_{g, a}=\mathrm{i} g \tanh (x / a), \text { with } g, a>0 .
$$

This PT-invariant potential of can be thought as an interpolation between the potential i $F x$ of an imaginary electric field $(g=a F, a \rightarrow+\infty)$ and the imaginary step potential $\operatorname{ig} \operatorname{sign}(x)$ (in the limit $a \rightarrow 0$ ). Define $H_{g, a}$ by (2) with the potential (8) on the domain of the second derivative. The solutions of the eigenvalue equation for (8) can be obtained by analytic continuation from those given in (Landau and Lifschitz 1977).

The operator $H_{g, a}$ has no eigenvalues. Its spectrum, which does not depend on $a$, is the union of two half-lines parallel to the real axis: $\{ \pm \mathrm{i} g+t ; t \geq 0\}$. In the imaginary electric field limit, the lines move to infinity, so that the spectrum of the limiting operator reduces to the point at infinity (see e.g. Herbst 1979).

Another instructive exactly soluble model is the $P T$-invariant "complexified harmonic oscillator" with the potential

$$
U_{\lambda}(x)= \begin{cases}\mathrm{e}^{\mathrm{i} \lambda} x^{2}, & \text { if } x>0 ; \\ \mathrm{e}^{-\mathrm{i} \lambda} x^{2}, & \text { if } x<0\end{cases}
$$

where $|\lambda| \leq \pi / 2$. For $\lambda=0$, (9) coincides the potential of the harmonic oscillator, while for $\lambda= \pm \pi / 2$ it is purely imaginary and antisymmetric, mimicking the cubic 
potential in (11). Let us also note that, in the limit $\lambda \rightarrow 0$ the perturbation (9) on the harmonic oscillator is somewhat milder than for the $\epsilon \rightarrow 0$ limit of the potential $-(\mathrm{i} x)^{2+\epsilon} \approx x^{2}\left[1+\epsilon \ln (|x|)+\mathrm{i} \frac{\epsilon \pi}{2} \operatorname{sign}(x)\right.$, considered by Bender and Boettcher(1998a).

The operator $H_{\lambda}$, defined on $D\left(\mathrm{~d}^{2} / \mathrm{d} x^{2}\right) \cap D\left(x^{2}\right)$, has compact (Hilbert-Schmidt) resolvent. But, for any $\lambda \neq 0$ only a finite number of the eigenvalues are real. The rest come in complex-conjugated pairs.

In the next section, we will compute exactly one value of the spectral zeta function $\left(Z_{H}(1)\right)$, the sum of the inverse eigenvalues, and will compare the result with numerical and WKB calculations for the real eigenvalues of (11), obtaining a remarkable coincidence. Since the real parts of any presumed complex eigenvalues must be positive, this places stringent bounds on the values of the maximum value of the ratio $\left|\operatorname{Re}\left(E_{\alpha}\right) / E_{\alpha}^{2}\right|$ for complex eigenvalues. In section 3 we will also show that the eigenfunctions (and their first derivatives) have no zeros on the real axis. This property holds for all potentials with monotonic imaginary part.

\section{Sum of inverse eigenvalues}

In our case we can set $g=1$ without altering generality. The eigenvalue equation (5) reads

$$
f^{\prime \prime}+\left(z-\mathrm{i} x^{3}\right) f=0 .
$$

Generally speaking, the solutions of (10) cannot be expressed in terms of the classical special functions. This is possible only for $z=0$ (Gradshtein and Ryzhik 1965, Caliceti et al 1980). Then, we can readily verify that

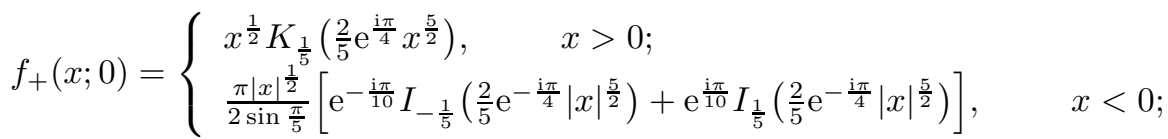

and

$$
f_{-}(x ; 0)=\overline{f_{+}(-x ; 0)},
$$

are a pair of PT-conjugated solutions of the Equation (10), and that $f_{+}(x ; 0)\left[f_{-}(x ; 0)\right]$ and their first derivatives are locally $\mathrm{L}^{2}$ near $+\infty[-\infty]$. Here, $I_{\nu}$ and $K_{\nu}$ denote the Bessel function of the first kind and imaginary argument, respectively Macdonald's function.

Although this is not immediately apparent from the representation (11), the solutions $f_{+}(x ; 0)$ and $f_{-}(x ; 0)$ are integer functions of $x$. The first terms of the McLaurin series for $f_{+}(x ; 0)$ are

$$
f_{+}(x ; 0)=\frac{\pi}{2 \sin \frac{\pi}{5}}\left[\frac{5^{-\frac{1}{5}} \mathrm{e}^{-\frac{\mathrm{i} \pi}{20}}}{\Gamma\left(\frac{4}{5}\right)}-\frac{5^{\frac{1}{5}} \mathrm{e}^{\frac{\mathrm{i} \pi}{20}}}{\Gamma\left(\frac{6}{5}\right)} x+\mathcal{O}\left(x^{5}\right)\right] .
$$

From (13) and (12) we get the $z=0$ value of the Wronskian (7)

$$
W\left[f_{+}, f_{-}\right](0)=-\frac{5 \pi}{4 \sin \frac{\pi}{10}} .
$$

The asymptotic behaviour of $f_{+}(x ; 0)$ near $\pm \infty$ is given by

$f_{+}(x ; 0) \approx \begin{cases}\frac{\sqrt{5 \pi}}{2 x^{\frac{3}{4}}} \exp \left(-\frac{2}{5} \mathrm{e}^{\frac{\mathrm{i} \pi}{4}} x^{\frac{5}{2}}-\frac{\mathrm{i} \pi}{8}\right)\left[1+\mathcal{O}\left(x^{-\frac{5}{2}}\right)\right], & x \rightarrow+\infty ; \\ \frac{\sqrt{5 \pi}}{|x|^{\frac{3}{4}} \sin \frac{\pi}{10}} \exp \left(\frac{2}{5} \mathrm{e}^{-\frac{\mathrm{i} \pi}{4}}|x|^{\frac{5}{2}}+\frac{\mathrm{i} \pi}{8}\right)\left[1+\mathcal{O}\left(|x|^{-\frac{5}{2}}\right)\right], & x \rightarrow-\infty .\end{cases}$ 
Using the asymptotic behaviour (15), Caliceti et al (1980) have shown that the kernel $G_{0}(x, y)$ belongs to the Hilbert-Schmidt class.

$$
\left\|G_{0}\right\|_{\mathrm{HS}}=\int_{-\infty}^{+\infty} \int_{-\infty}^{+\infty} \mathrm{d} x \mathrm{~d} y\left|G_{0}(x, y)\right|^{2}<\infty .
$$

Here we will note that the diagonal elements of the continuous in $(x, y)$ Hilbert-Schmidt kernel $G_{0}(x, y)$ is absolutely summable,

$$
\int_{-\infty}^{+\infty} \mathrm{d} x\left|G_{0}(x, x)\right|<\infty
$$

since the continuous integrand is summable near both infinities,

$$
\left|f_{+}(x ; 0) f_{-}(x ; 0)\right| \approx \mathcal{O}\left(|x|^{-3 / 2}\right), \text { when } x \rightarrow \pm \infty .
$$

The trace is equal to the sum of all the inverse eigenvalues

$$
Z_{H}(1)=\sum_{j} \frac{1}{E_{j}}=-\int_{-\infty}^{+\infty} \mathrm{d} x G_{0}(x, x),
$$

which is finite. Analogous formulae can be written for the zeta functions of integer arguments $s>1$. Taking into account the PT-conjugacy of $f_{+}$and $f_{-}$, we obtain

$$
\begin{aligned}
Z_{H}(1)=\frac{2 \sin \frac{\pi}{10}}{5 \pi} \operatorname{Re}\left[\int_{0}^{+\infty} \mathrm{d} x f_{+}(x ; 0) f_{-}(x ; 0)\right]= \\
\left(\frac{2}{5}\right)^{\frac{6}{5}} \int_{0}^{+\infty} \mathrm{d} t t^{-\frac{1}{5}} K_{\frac{1}{5}}(t)\left[I_{-\frac{1}{5}}(t)+\frac{\cos (3 \pi / 10)}{\cos (\pi / 10)} I_{\frac{1}{5}}(t)\right]= \\
\frac{\Gamma^{2}\left(\frac{1}{5}\right)}{5^{6 / 5} \Gamma\left(\frac{3}{5}\right)}\left[3-2 \cos \frac{\pi}{5}\right] \approx 2.835094933 .
\end{aligned}
$$

In the last integral we rotated the contour of integration by $-\pi / 10$, making the substitution $x=(5 t / 2)^{\frac{2}{5}} \mathrm{e}^{-\mathrm{i} \pi / 10}$, which leads to tabulated integrals (Gradshtein and Ryzhik 1965, 6.576.5). (10)

Bender and Boettcher (1998a) gave a WKB estimate for the large eigenvalues of

$$
E_{j} \approx E_{j}^{(q c)}=\left[5 \sqrt{\frac{\pi}{3}} \frac{\Gamma\left(\frac{5}{6}\right)}{\Gamma\left(\frac{1}{3}\right)}\left(j+\frac{1}{2}\right)\right]^{6 / 5} .
$$

Comparison of (21) with the numerical results of Bender and Boettcher (1998a) shows that even for $j=0$ the error is about $5 \%$.

The sum of all inverse WKB eigenvalues (21) can be expressed in terms of the Riemann zeta function:

$Z_{H}^{(q c)}(1)=\sum_{j} \frac{1}{E_{j}^{(q c)}}=\left[5 \sqrt{\frac{\pi}{3}} \frac{\Gamma\left(\frac{5}{6}\right)}{\Gamma\left(\frac{1}{3}\right)}\right]^{6 / 5}\left(2^{\frac{6}{5}}-1\right) \zeta\left(\frac{6}{5}\right) \approx 2.885673793$.

We can correct the WKB approximation (22) for $Z_{H}(1)$ by taking the numerical values of the first five eigenvalues from Table 1 of Bender and Boettcher (1998a), 
who give their result to five digit precision. This yields 2.8351 , to be compared with 2.835094933 from (20). A remarkable coincidence! The two values coincide within the five-digit acuracy of the numerical eigenvalues. Since the real parts of any presumed complex eigenvalues must be positive, no compensations can occur in the sum. If some complex conjugate eigenvalues $E_{\alpha}$ are present, then the largest ratio

$$
\max _{\alpha}\left|\frac{\operatorname{Re}\left(E_{\alpha}\right)}{E_{\alpha}^{2}}\right|<10^{-5} .
$$

We consider this as strongly supportive for the conjectured (Bessis 1995) absence of complex eigenvalues.

\section{Nodeless character of the eigenfunctions}

As we can see from (21), the sum (19) converges slowly. An improvement in the precision of the comparison between (20) and numerical result swill require precision computations of many eigenvalues. Bender and Boettcher (1998a) integrated the differential equation along the anti-Stokes lines in the lower complex half-plane, starting with large values of $|x|$, where the asymptotic behavior is attained, and matching the solutions at $x=0$. The following proposition on the properties of the eigenfunctions could prove useful for simplifying numerical work.

Let $\phi_{E}$ be an eigenfunction corresponding to the eigenvalue $z=E$ in (10). Then, proceeding as in the case of proving current conservation for the solutions of the stationary Schrödinger equation, we obtain

$$
j_{E}^{\prime}(x)=\operatorname{Im}\left[\phi_{E}^{\prime}(x) \overline{\phi_{E}(x)}\right]^{\prime}=\left[x^{3}-\operatorname{Im}(E)\right]\left|\phi_{E}(x)\right|^{2} .
$$

The current density associated to an eigenfunction is a decreasing (increasing) function of $x$ if $x^{3}-\operatorname{Im}(E)<(>) 0$. Since the limit $j_{E}( \pm \infty)=0$, the current density is strictly negative on $(-\infty,+\infty)$ and has a unique minimum at $x=\sqrt[3]{\operatorname{Im}(E)}$. Thus,

a. The eigenfunctions of (10) have no zeros on the real axis;

b. The imaginary part of their logarithmic derivatives, $\operatorname{Im}\left[\phi_{E}^{\prime}(x) / \phi_{E}(x)\right]$, have constant sign.

This proposition can be readily generalized to the eigenfunctions of Equation (2) with a complex potential $U(x)$ having a monotonic imaginary part.

Using the above proposition we can look for the eigenvalues of (10) by a shooting method, integrating the Ricatti equation satisfied by the logarithmic derivative $s(x)=f^{\prime}(x) / f(x)$

$$
s^{\prime}+s^{2}+E-\mathrm{i} x^{3}=0 .
$$

In the selfadjoint case, the eigenfunctions have nodes on the real axis, which leads to singularities in the logarithmic derivative. Thus, although the order of the equation can be lowered, this is not quite helpful for numerical calculations. In our case, the eigenfunctions corresponding to real eigenvalues are PT-symmetric (eventually up to an irrelevant phase factor). This implies that if $s(x ; E)$ is the logarithmic derivative of an eigenfunction, then $s(0 ; E)$ is purely imaginary. The large $x$ asymptotic behaviour of the solution of (25) whose real part goes to $-\infty$ as $x \rightarrow+\infty$ is

$$
s(x) \approx-\frac{1+\mathrm{i}}{\sqrt{2}} x^{\frac{3}{2}}-\frac{3}{4 x}+\frac{1-\mathrm{i}}{2 \sqrt{2}} E x^{-\frac{3}{2}}+\mathcal{O}\left(x^{-\frac{7}{2}}\right) ; \quad x \rightarrow+\infty .
$$


Now, choosing a sufficiently large $X$, where $s(X)$ for the solution which decays at infinity is close to the asymptotic, we can integrate (25) from $X$ backwards to $x=0$. If $E$ is an eigenvalue, then

$$
\operatorname{Re}[s(0 ; E)]=0 .
$$

High precision solutions of (27) could be obtained by a simple Newton routine, starting at the WKB prediction (21).

The advantage of this approach is that we integrate a complex first order equation instead of the original second order one. The requirement that the imaginary part of $s$ is negative allows stopping the integration if we detect a change of $\operatorname{sign}$ in $\operatorname{Im}(s, E)$. That energy cannot be an eigenvalue. If $X$ is sufficiently large, the eigenvalues are relatively insensitive to small perturbations of the boundary condition at $X$. Practical coonsiderations require us to start with the lowest value of $X$, where the decaying solution is sufficiently close to the asymptotic behaviour (26). An alternative approach for the large eigenvalues, would be to obtain corrections to the leading order WKB result (21).

\section{Acknowledgments}

Partial support by the Academy of Finland under Project No 163394 is gratefully acknowledged. I thank Masud Chaichian for hospitality at the Department of High Energy Physics, Helsinki University, where part of this work has been done, and Daniel Bessis for discussions.

\section{References}

Akhiezer N I and Glazman I M 1966 Theory of Linear Operators in Hilbert Space 2nd edition (Moscow: Nauka) (in Russian); English translation 1993 (New-York: Dover)

Bender C M and Boettcher S 1998a Phys. Rev. Lett. 805243

-1998b J. Phys. A: Math. Gen. 31 L273

Bender C M, Boettcher S and Meisinger P N 1999 J. Math. Phys. 402201

Bessis D 1995 Private communication.

Caliceti E, Graffi S and Maioli M 1980 Commun. Math. Phys. 7551

Caliceti E 1999 Distributional Borel summability of odd anharmonic oscillators Preprint mp_arc 99-384; http://www.math.utexas.edu

Delabaere E and Pham F 1998 Phys. Lett. A250 25

Gradshtein I S and Ryzhik I M 1965 Tables of Integrals, Series and Products 4th edition (NewYork: Academic Press)

Herbst I 1979 Commun. Math. Phys. 64279

Kato T 1966 Perturbation Theory for Linear Operators (Berlin: Springer)

Landau L D and Lifschitz E M 1977 Quantum Mechanics 3rd edition (Oxford: Pergamon)

Naimark M A 1964 Linear Differential Operators Part II (London: Harrap)

Reed M and Simon B 1972 Methods of Modern Mathematical Physics Vol. 1 (New York: Academic Press) 